\title{
The influence of the reclassification of NIFTP as an uncertain tumour on risk of malignancy for the diagnostic categories according to the Bethesda system for reporting thyroid cytopathology
}

\author{
Janusz Kopczyński ${ }^{1}$, Agnieszka Suligowska², Kornelia Niemyska', Iwona Pałyga ${ }^{2}$, Agnieszka Walczyk², \\ Danuta Gąsior-Perczak², Artur Kowalik³, Kinga Hińcza ${ }^{3}$, Ryszard Mężyk², Stanisław Góźdźż, 5 , \\ Aldona Kowalska ${ }^{2,5}$
}

${ }^{1}$ Department of Pathology, Holycross Cancer Centre, Kielce, Poland

${ }^{2}$ Endocrinology Clinic of Holycross Cancer Centre, Kielce, Poland

${ }^{3}$ Department Molecular Diagnostics, Holycross Centre, Kielce, Poland

${ }^{4}$ Oncology Clinic, Holycross Cancer Centre, Kielce, Poland

${ }^{5}$ The Faculty of Health Sciences of the Jan Kochanowski University, Kielce, Poland

\begin{abstract}
Introduction: The noninvasive encapsulated, follicular variant of papillary thyroid carcinoma was reclassified as noninvasive follicular thyroid neoplasm with papillary-like nuclear features (NIFTP). The exclusion of NIFTP from the group of malignant tumours decreases the risk of malignancy (RoM) as defined by the Bethesda System for Reporting Thyroid Cytopathology (TBSRTC). The aim of the present study was to evaluate the RoM for each category in TBSRTC with and without exclusion of NIFTP from the tally of malignancies. Material and methods: The present study included 998 thyroid nodules cases. All patients underwent diagnostic tests, including fine-needle aspiration cytology, and received surgical treatment. Slides for all resection specimens with a diagnosis of cancer were reviewed to identify NIFTP. The RoM for each of the categories in TBSRTC with and without exclusion of NIFTP from the malignant tumours was evaluated. Results: The RoM decreased with the exclusion of NIFTP from malignant categorisation with the following values for the different TBSRTC categories: non-diagnostic (ND): $0 \%$; benign: 0\%; atypia/follicular lesion of undetermined significance (AUS/FLUS): $1.6 \%$; follicular neoplasm/suspicious for follicular neoplasm (FN/SFN): $0.7 \%$; suspicious for malignancy (SUS): $6.9 \%$; and malignant: $2.5 \%$. The difference of $2.5 \%$ in the malignant category was statistically significant $(\mathrm{p}=0.0253)$.

Conclusions: The RoM for specific TBSRTC categories needs to be defined for each treatment centre because it is important for the selection of the appropriate surgical treatment for thyroid tumours. (Endokrynol Pol 2019; 70 (3): 232-236)
\end{abstract}

Key words: noninvasive follicular thyroid neoplasm with papillary-like nuclear features (NIFTP); risk of malignancy (RoM); thyroid cancer; Bethesda system

\section{Introduction}

The incidence of thyroid cancer (TC) has increased in the last few decades [1-3], with papillary thyroid carcinoma (PTC) of low clinical grade accounting for the majority of cases [4].

The frequency of encapsulated follicular tumours, which are characterised by highly indolent behaviour, is $22-34 \%$ of all PTCs and is becoming exceedingly high $[5,6]$. However, the mortality of PTC remains low [3]. Therefore, overtreatment of PTC is an issue of concern [7]. In 2015, the Endocrine Pathology Society working group included experts from the interdisciplinary research team reclassified the noninvasive, encapsulated, follicular variant of PTC as noninvasive follicular thyroid neoplasm with papillary-like nuclear features (NIFTP) [8]. The proposed changes were accepted by the American Thyroid Association (ATA), and new diagnostic criteria and treatment methods were included in the association's recommendations [9]. The treatment of NIFTP consists of surgical removal of the thyroid lobe involved without the need for total resection or lymph node resection. Adjuvant therapy with radioiodine or suppressive doses of thyroxine is not indicated [9]. The diagnosis of NIFTP is confirmed after surgery, and these tumours cannot be diagnosed based on the results of fine-needle aspiration cytology (FNAC). According to the cytology results, NIFTP is frequently 
classified as category III, IV, V, and VI according to the Bethesda system for reporting thyroid cytopathology (TBSRTC) [10, 11]. The exclusion of NIFTP from the group of malignant tumours decreases the risk of malignancy (RoM) in specific TBSRTC categories. Studies addressing this issue report considerable differences in the incidence of NIFTP and variation in the RoM in specific TBSRTC categories among different centres [12-20]. Numerous factors may impact differences between various centres. One of them is the criteria for the identification of a given category, especially the one that evokes the most controversy - category III according to the TBSRTC. AUS/FLUS is identified when a lesion does not meet the qualitative or quantitative criteria for category IV or V, and this is a diagnosis of exclusion. By contrast, with category IV the sample may exhibit: sheets of follicular cells, colloid in background, and foamy macrophages. Moreover, the assignment to category III may also result from limitation of a given sample, such as: low cellularity, blood admixture, and incorrect fixation [21]. AUS/ FLUS is a temporary diagnosis that requires verification in correlation with the clinical and a repeated FNAC. It is not an indication for surgery unless, as in our work, there are clinical or ultrasonographic signs of a risk of malignancy. The level of iodine supplementation in a given society is another factor. Owing to iodisation of salt, Poland is one of the countries with effective iodine prophylaxis. However, it is estimated that insufficient dietary iodine intake concerns 52\% of European citizens. For comparison, this level reaches 11\% in North and South America [22, 23]. Iodine deficiency leads to the development of toxic or neutral nodular goitre and, in severe cases, to hypothyroidism. Moreover, in the context of this study, it is significant to note that regions with iodine deficiency are characterised by greater incidence of follicular thyroid carcinoma compared with papillary carcinoma, which is associated with less frequent occurrence of NIFTP. These differences suggest that individual analysis and evaluation are necessary. The aim of the present study was to evaluate the RoM in specific TBSRTC categories with and without the exclusion of NIFTP from the tally of malignancies. The study was conducted in a single centre in which patients are diagnosed and surgically treated. The authors had unlimited access to patients' medical records, as well as FNAC results, cytological slides, and paraffin blocks from surgical material.

\section{Material and methods}

A search of the pathology database was performed to identify all thyroidectomy specimens resected between January 2000 and December 2015. For each case all surgical pathology reports were reviewed. For each surgical specimen, the preceding FNAC reports were identified.
The fine-needle aspiration cytology was performed by a cytopathologist under ultrasound supervision. The location of the tumour according to the ultrasound results and histopathology was compared and documented. Thyroid tumours that were found incidentally and were not diagnosed in advance (17 cases) were excluded from the study.

The results of FNAC performed before introducing TBSRTC were re-evaluated by a cytopathologist, and the tumours were classified into the appropriate categories according to the TBSRTC [11]. The histopathological materials of all the cases classified as malignant were re-evaluated according to the new definition of NIFTP as an uncertain tumour $[8,24]$. The RoM for each category in TBSRTC with and without exclusion of NIFTP from the malignant tumours was evaluated. For patients with FNACs of multiple nodules, only the nodule and corresponding FNAC associated with the highest risk of malignancy was evaluated, with the risk of malignancy based on statistics quoted for the Bethesda system. The $\chi^{2}$ test was used to evaluate the statistical significance of variables. The study plan was accepted by the Bioethics Committee at the Regional Chamber of Physicians without the necessity to obtain the patients' written informed consent because the data obtained was retrospective data from the patients' medical history, which that was carried out during routine diagnostic procedures while hospitalised. All patients' records information were anonymised and de-identified prior to analysis.

\section{US-guided FNAC technique}

Informed consent is obtained after the biopsy purpose and procedure are discussed with the patient. The fine-needle aspiration cytology is performed using a 27-gauge needle under ultrasound guidance to ensure accurate placement of the needle within the thyroid nodule. Before aspiration, scanning is performed in the transverse plane for lesion localisation, followed by colour Doppler mapping to depict any large blood vessels in and around the nodule, so that vascular injury can be avoided during the procedure. The patient is instructed to remain as still as possible and avoid coughing, talking, and swallowing during the biopsy. A freehand biopsy technique is used, and the syringe attached to the needle is placed just above the ultrasound probe. The needle is guided parallel or perpendicular to the probe, and the needle tip is carefully monitored during the procedure. When the needle reaches the target, the biopsy is performed. Aspiration is performed at least twice. Cytological slides are prepared by dispensing the aspirated materials from the needle onto glass slides and compressing them with a second slide; samples are then immediately fixed with 95\% ethyl alcohol and stained using the Papanicolaou method.

\section{Results}

Our cohort included 998 consecutive patients. There were $860(86.2 \%)$ women and $138(13.8 \%)$ men, and the average age of the patients was $51 \pm 13$ years. The results of FNAC according to TBSRTC are presented in Table I. According to the postoperative histopathological diagnosis, a benign tumour was diagnosed in 725 patients (72.6\%) and a malignant tumour was diagnosed in 273 patients $(27.4 \%)$. Among the malignant tumours, 214 (78.3\%) were papillary carcinoma, 13 (4.8\%) were follicular carcinoma, 30 (10.9\%) were medullary carcinoma, eight $(2.9 \%)$ were poorly differentiated cancer, five $(1.9 \%)$ were anaplastic cancer, two $(0.8 \%)$ were lymphoma, and one $(0.4 \%)$ was angiosarcoma. Data on cancer types corresponding to particular diagnostic categories are shown in Table II. The RoM values for each specific TBSRTC category are presented in Table I and were as follows: 
Table I. Comparison of risk of malignancy with and without the NIFTP category

\begin{tabular}{lccccccc}
\hline $\begin{array}{l}\text { TBSRTC } \\
\text { diagnostic } \\
\text { categories }\end{array}$ & Total no. & $\begin{array}{c}\text { No malignancy } \\
\text { with NIFTP }\end{array}$ & $\begin{array}{c}\text { RoM with } \\
\text { NIFTP }\end{array}$ & $\begin{array}{c}\text { No malignancy } \\
\text { without NIFTP }\end{array}$ & $\begin{array}{c}\text { RoM without } \\
\text { NIFTP }\end{array}$ & $\begin{array}{c}\text { Difference (95\% } \\
\text { CI) }\end{array}$ & p-value \\
\hline ND & $6(0.6)$ & $1(16.7)$ & $16.7 \%$ & $1(16.7)$ & $16.7 \%$ & $0 \%(-42.0-42.0)$ & 1.0000 \\
\hline Benign & $522(52.3)$ & $6(1.2)$ & $1.2 \%$ & $6(1.2)$ & $1.2 \%$ & $0 \%(-1.5-1.5)$ & 1.0000 \\
\hline AUS/FLUS & $62(6.2)$ & $7(11.3)$ & $11.3 \%$ & $6(9.7)$ & $9.7 \%$ & $1.6 \%(-9.8-13.1)$ & 0.7723 \\
\hline FN/SFN & $138(13.8)$ & $18(13.0)$ & $13.0 \%$ & $17(12.3)$ & $12.3 \%$ & $0.7 \%(-7.3-8.7)$ & 0.8614 \\
\hline SUS & $72(7.2)$ & $43(59.7)$ & $59.7 \%$ & $38(52.8)$ & $52.8 \%$ & $6.9 \%(-9.1-22.4)$ & 0.4056 \\
\hline Malignant & $198(19.9)$ & $198(100)$ & $100 \%$ & $193(97.5)$ & $97.5 \%$ & $2.5 \%(0.1-5.7)$ & 0.0253 \\
\hline Total & $998(100)$ & $273(27.4)$ & $27.4 \%$ & $261(26.1)$ & $26.1 \%$ & $1.3 \%(-2.6-5.2)$ & 0.5119 \\
\hline
\end{tabular}

RoM — risk of malignancy; TBSRTC — Bethesda system for reporting thyroid cytopathology; ND — non-diagnostic; AUS/FLUS — atypia of undetermined significance/ /follicular lesion of undetermined significance; FN/SFN — follicular neoplasm/suspicious of follicular neoplasm; SUS — suspicious of malignancy; NIFTP — noninvasive follicular thyroid neoplasm with papillary-like nuclear features

Table II. Types of cancers in particular diagnostic categories according to the Bethesda system for reporting thyroid cytopathology

\begin{tabular}{|c|c|c|c|c|c|c|c|}
\hline $\begin{array}{l}\text { TBSRTC } \\
\text { diagnostic } \\
\text { categories }\end{array}$ & $\begin{array}{l}\text { Papillary } \\
\text { carcinoma }\end{array}$ & $\begin{array}{l}\text { Follicular } \\
\text { carcinoma }\end{array}$ & $\begin{array}{l}\text { Medullary } \\
\text { carcinoma }\end{array}$ & $\begin{array}{c}\text { Poorly } \\
\text { differentiated } \\
\text { cancer }\end{array}$ & $\begin{array}{c}\text { Anaplastic } \\
\text { cancer }\end{array}$ & Lymphoma & Angiosarcoma \\
\hline ND & 1 & 0 & 0 & 0 & 0 & 0 & 0 \\
\hline Benign & 3 & 2 & 1 & 0 & 0 & 0 & 0 \\
\hline AUS/FLUS & 3 & 3 & 1 & 0 & 0 & 0 & 0 \\
\hline FN/SFN & 14 & 4 & 0 & 0 & 0 & 0 & 0 \\
\hline SUS & 31 & 1 & 3 & 4 & 1 & 2 & 1 \\
\hline Malignant & 162 & 3 & 25 & 4 & 4 & 0 & 0 \\
\hline Total & 214 (78.3\%) & $13(4.8 \%)$ & $30(10.9 \%)$ & $8(2.9 \%)$ & $5(1.9 \%)$ & $2(0.8 \%)$ & $1(0.4 \%)$ \\
\hline
\end{tabular}

TBSRTC — Bethesda system for reporting thyroid cytopathology; ND — non-diagnostic; AUS/FLUS — atypia of undetermined significance/follicular lesion of undetermined significance; FN/SFN — follicular neoplasm/suspicious of follicular neoplasm; SUS — suspicious of malignancy

ND - 16.7\%; benign - 1.2\%; AUS/FLUS - 11.3\%; FN/SFN - 13.0\%; SUS - 59.7\%; malignant - 100\%. Based on the re-evaluation of histopathology results, 12 cases were diagnosed as NIFTP, which accounted for $5.7 \%$ of all papillary carcinoma diagnoses. Cytological materials from NIFTP cases were classified into AUS/FLUS in one case, FN/SFN in one case, SUS in five cases, and malignant in five cases. The RoM values for each specific TBSRTC category after NIFTP exclusion were as follows: ND - 16.7\%; benign - 1.2\%; AUS/FLUS - 9.7\%; FN/SFN - 12.3\%; SUS - 52.8\%; malignant — $97.5 \%$ The RoM decreased with the exclusion of NIFTP from malignant categorisation with the following values for the different TBSRTC categories: ND $-0 \%$; benign $-0 \%$; AUS/FLUS - $1.6 \%$; FN/SFN - 0.7\%; SUS - 6.9\%; malignant $-2.5 \%$.The difference of $2.5 \%$ in the malignant category was statistically significant $(p=0.0253)($ Tab. I).

\section{Discussion}

In recent years, the overtreatment of TC has become an issue of concern [4, 7]. The risk of side effects (hy- poparathyroidism, vocal cord paralysis, or potential side effects of isotope therapy), psychological damage as a result of the cancer diagnosis, and social costs associated with the treatment and prolonged follow-up are frequently raised issues [26-28].

The exclusion of NIFTP with a good prognosis from the group of malignant tumours contributed to improving the current situation. Approximately 45,000 patients are diagnosed with NIFTP each year worldwide [8]. This relatively large group of patients do not receive a cancer diagnosis because of the reclassification, thus preventing unnecessary aggressive treatment and the stress associated with the diagnosis itself, as well as avoiding medical appointments. Along with the reduction in the number of malignant tumours by the number of cases diagnosed as NIFTP, the estimated RoM in particular TBSRTC categories decreases as well.

Different centres reported a variable reduction in the RoM. In the present study, the greatest reductions in RoM were observed in SUS, malignant, and AUS/FLUS. In the ND category, the RoM remained unchanged after the introduction of the new NIFTP classification. How- 
Table III. Decrease in the risk of malignancy in the Bethesda System for Reporting Thyroid Cytopathology categories according to the inclusion of NIFTP as a non-malignant tumour

\begin{tabular}{|c|c|c|c|c|c|c|}
\hline \multirow{2}{*}{ Study } & \multicolumn{6}{|c|}{ Percentage decrease in the RoM of TBSRTC categories } \\
\hline & ND & Benign & AUS/FLUS & FN/SFN & SUS & Malignant \\
\hline Current study & 0 & 0 & 1.6 & 0.7 & 6.9 & 2.5 \\
\hline $\begin{array}{l}\text { Zhou [13] } \\
\text { Institution A }\end{array}$ & 0 & 2.5 & 15 & 11.2 & 26.8 & 3.0 \\
\hline $\begin{array}{l}\text { Zhou [13] } \\
\text { Institution B }\end{array}$ & 0 & 0.8 & 7.3 & 6.4 & 7.8 & 4.4 \\
\hline $\begin{array}{l}\text { Zhou [13] } \\
\text { Institution C }\end{array}$ & 0 & 0 & 5.2 & 3.9 & 0 & 0.4 \\
\hline Faquin 2016 [14] & 1.4 & 3.5 & 13.6 & 15.1 & 23.4 & 3.3 \\
\hline Strickland 2015 [15] & 1.9 & 7.8 & 17.6 & 8.0 & 41.5 & 5.1 \\
\hline Lau 2017 [16] & 0 & 3.0 & 20.0 & 30,8 & 32.0 & 3.0 \\
\hline Range 2017 [20] & 0.0 & 0.0 & 5.0 & 2.0 & 24.0 & 0.0 \\
\hline Li 2017 [19] & 0.0 & 1.2 & 3.3 & 5.9 & 2.3 & 0.6 \\
\hline Canberk 2016 [17] & 6.5 & 1.0 & 15 & 20 & 24 & 11 \\
\hline Layfield 2017 [18] & 0.0 & 3.6 & 2.3 & 2.5 & 17.0 & 12.8 \\
\hline Kiernan 2017 [28] & 0.0 & 0.2 & 2.0 & 2.0 & 8.0 & 1.0 \\
\hline
\end{tabular}

RoM — risk of malignancy; TBSRTC — Bethesda system for reporting thyroid cytopathology; ND — non-diagnostic; AUS/FLUS — atypia of undetermined significance/ /follicular lesion of undetermined significance; FN/SFN — follicular neoplasm/suspicious of follicular neoplasm; SUS — suspicious of malignancy; NIFTP — noninvasive follicular thyroid neoplasm with papillary-like nuclear features

ever, the RoM decreased maximally by $6.5 \%$ according to a study by Canberk (Tab. III).

Similarly, in the benign category, the RoM remained unchanged after the exclusion of NIFTP from the tally of malignancies. The greatest change in the RoM value in this category was reported by Strickland, who showed that the introduction of NIFTP reduced the RoM by $7.8 \%$ [15]. The results reported by two other centres were similar to the present data, with no changes observed in the RoM in this category [13, 20] (Tab. III).

In the present study, the RoM in AUS/FLUS was reduced by $1.6 \%$, and this was the smallest difference observed in centres analysing RoM variation after NIFTP exclusion. The greatest variations in this category were observed by Strickland (17.6\%) and Lau (20\%). In FN/SFN, a 2\% difference in the RoM was reported by Range and $30.8 \%$ was reported by Lau (Tab. III). In the present study the RoM was reduced by $0.7 \%$.

In the present study, the SUS category showed the greatest reduction in the RoM, at $6.9 \%$; in other centres it ranged from $0 \%$ [13] to $41 \%$ [15]. In the malignant category, the change in the NIFTP classification reduced the RoM by $2.5 \%$, from $100 \%$ to $97.5 \%$ (Table III). Layfield reported a reduction in the RoM of $12.8 \%$ according to the new NIFTP classification, whereas Canberk reported a reduction of 11\% [17], both of which are considerable reductions in the RoM values compared with previous data. In the present study, the RoM for the malignant category remained high even after the exclusion of NIFTP from the group of malignant tumours. This facilitates the determination of the appropriate surgical approach as in the case of malignant tumours.

Because of significant differences in RoM between different centres, defining the RoM value in the TBSRTC category is crucial in each centre, because it facilitates the selection of appropriate surgical treatments based on the FNAC results.

\section{Conclusions}

The introduction of TBSRTC resulted in a unified system of evaluating cytological material and the establishment of the RoM for particular categories, leading to the standardisation of treatments according to the FNAC results. The new NIFTP classification reduces the RoM in most TBSRTC categories to different extents in the different reporting centres. In the present study only, the difference in category VI was statistically significant. In the remaining categories, the differences were relatively small and not statistically significant. Definition of the RoM in each specific TBSRTC category in each centre is crucial for planning the appropriate surgical treatment of thyroid tumours.

\section{Conflict of interest}

The authors declare that they have no conflict of interest. 


\section{Funding}

The study was funded by Holycross Cancer Centre. The funder had no role in the study design, data collection and analysis, decision to publish, or preparation of the manuscript.

\section{References}

1. Lim H, Devesa SS, Sosa JA, et al. Trends in thyroid cancer incidence and mortality in the United States, 1974-2013. JAMA. 2017; 317(13): 1338-1348, doi: 10.1001/jama.2017.2719, indexed in Pubmed: 28362912.

2. Davies L, Welch HG. Increasing incidence of thyroid cancer in the United States, 1973-2002. JAMA. 2006; 295(18): 2164-2167, doi: 10.1001/jama.295.18.2164, indexed in Pubmed: 16684987.

3. La Vecchia C, Malvezzi M, Bosetti C, et al. Thyroid cancer mortality and incidence: a global overview. Int J Cancer. 2015; 136(9): 2187-2195, doi: 10.1002/ijc.29251, indexed in Pubmed: 25284703.

4. Ahn HS, Kim HJ, Welch HG. Korea's thyroid-cancer "epidemic" — screening and overdiagnosis. N Engl J Med. 2014; 371(19): 1765-1767, doi: 10.1056/NEJMp1409841, indexed in Pubmed: 25372084.

5. Jung CK, Little MP, Lubin JH. The increase in thyroid cancer incidence during the last four decades is accompanied by a high frequency of BRAF mutations and a sharp increase in RAS mutations. J Clin Endocrinol Metab. 2014; 99(1): 276-284, doi: 10.1210/jc.2013-2503, indexed in Pubmed: 24248188 .

6. Lin HW, Bhattacharyya N. Clinical behavior of follicular variant of papillary thyroid carcinoma: presentation and survival. Laryngoscope. 2010; 120(4): 712-716, doi: 10.1002/lary.20828, indexed in Pubmed: 20205241.

7. Welch HG, Black WC. Overdiagnosis in cancer. J Natl Cancer Inst. 2010; 102(9): 605-613, doi: 10.1093/jnci/djq099, indexed in Pubmed: 20413742.

8. Nikiforov YE, Seethala RR, Tallini G, et al. Nomenclature revision for encapsulated follicular variant of papillary thyroid carcinoma: a paradigm shift to reduce overtreatment of indolent tumors. JAMA Oncol. 2016; 2(8): 1023-1029, doi: 10.1001/jamaoncol.2016.0386, indexed in Pubmed: 27078145.

9. Haugen BR, Sawka AM, Alexander EK, et al. American Thyroid Association Guidelines on the Management of Thyroid Nodules and Differentiated Thyroid Cancer Task Force Review and Recommendation on the Proposed Renaming of Encapsulated Follicular Variant Papillary Thyroid Carcinoma Without Invasion to Noninvasive Follicular Thyroid Neoplasm with Papillary-Like Nuclear Features. Thyroid. 2017; 27(4): 481-483, doi: 10.1089/thy.2016.0628, indexed in Pubmed: 28114862.

10. Cibas ES, Ali SZ. The 2017 Bethesda System for Reporting Thyroid Cytopathology. Thyroid. 2017; 27(11): 1341-1346, doi: 10.1089/thy.2017.0500, indexed in Pubmed: 29091573.

11. Cibas ES, Ali SZ. The Bethesda System for reporting thyroid cytopathology. Thyroid. 2009; 19(11): 1159-1165, doi: 10.1089/thy.2009.0274, indexed in Pubmed: 19888858.

12. Fisher SB, Perrier ND. The incidental thyroid nodule. CA Cancer J Clin. 2018; 68(2): 97-105, doi: 10.3322/caac.21447, indexed in Pubmed: 29369334.

13. Zhou H, Baloch ZW, Nayar R, et al. Noninvasive follicular thyroid neoplasm with papillary-like nuclear features (NIFTP): Implications for the risk of malignancy (ROM) in the Bethesda System for Reporting Thyroid Cytopathology (TBSRTC). Cancer Cytopathol. 2018; 126(1): 20-26, doi: 10.1002/cncy.21926, indexed in Pubmed: 28941185.

14. Faquin WC, Wong LQ, Afrogheh AH, et al. Impact of reclassifying noninvasive follicular variant of papillary thyroid carcinoma on the risk of malignancy in The Bethesda System for Reporting Thyroid Cytopathology. Cancer Cytopathol. 2016; 124(3): 181-187, doi: 10.1002/cncy.21631, indexed in Pubmed: 26457584.

15. Strickland KC, Howitt BE, Marqusee E, et al. The impact of noninvasive follicular variant of papillary thyroid carcinoma on rates of malignancy for fine-needle aspiration diagnostic categories. Thyroid. 2015; 25(9): 987-992, doi: 10.1089/thy.2014.0612, indexed in Pubmed: 26114752.

16. Lau RP, Paulsen JD, Brandler TC, et al. Impact of the Reclassification of "Noninvasive Encapsulated Follicular Variant of Papillary Thyroid Carcinoma" to "Noninvasive Follicular Thyroid Neoplasm With Papillary-Like Nuclear Features" on the Bethesda System for Reporting Thyroid Cytopathology: A Large Academic Institution's Experience. Am J Clin Pathol. 2017; 149(1): 50-54, doi: 10.1093/ajcp/aqx136, indexed in Pubmed: 29272354.

17. Canberk S, Gunes P, Onenerk M, et al. New concept of the encapsulated follicular variant of papillary thyroid carcinoma and its impact on the Bethesda System for reporting thyroid cytopathology: a single-institute experience. Acta Cytol. 2016; 60(3): 198-204, doi: 10.1159/000447990, indexed in Pubmed: 27414983.

18. Layfield LJ, Baloch ZW, Esebua M, et al. Impact of the reclassification of the non-invasive follicular variant of papillary carcinoma as benign on the malignancy risk of the Bethesda System for reporting thyroid cytopathology: a meta-analysis study. Acta Cytol. 2017; 61(3): 187-193, doi: 10.1159/000469654, indexed in Pubmed: 28445876.

19. Li WSB, Pang J, Lew M, et al. Implementing NIFTP may potentially impact the risk of malignancy for thyroid nodules categorized as FLUS and SFN. Mod Pathol. 2017; 30: 104A.

20. Range DEHG, Jiang X. The impact of reclassification of encapsulated follicular variant of papillary thyroid carcinoma on the risk of malignancy in the Bethesda System for Reporting of Thyroid Cytopathology. Mod Pathol. 2017; 30: 147A.

21. Jarząb B, Dedecjus M, Słowińska-Klencka D, et al. Guidelines of Polish National Societies Diagnostics and Treatment of Thyroid Carcinoma. 2018 Update. Endokrynol Pol. 2018; 69(1): 34-74, doi: 10.5603/EP.2018.0014, indexed in Pubmed: 29442352.

22. de Benoist B, McLean E, Andersson M, et al. Iodine deficiency in 2007: global progress since 2003. Food Nutr Bull. 2008; 29(3): 195-202, doi: 10 $.1177 / 156482650802900305$, indexed in Pubmed: 18947032

23. Gietka-Czernel M. Iodine prophylaxis. Post Nauk Med. 2015; 12: 839-845.

24. Kakudo K, El-Naggar AK, Hodak SP, et al. Noninvasive follicular thyroid neoplasm with papillary-like nuclear features (NIFTP) in thyroid tumor classification. Pathol Int. 2018; 68(6): 327-333, doi: 10.1111/pin.12673, indexed in Pubmed: 29675873.

25. Marti JL, Jain KS, Morris LGT. Increased risk of second primary malignancy in pediatric and young adult patients treated with radioactive iodine for differentiated thyroid cancer. Thyroid. 2015; 25(6): 681-687, doi: 10.1089/thy.2015.0067, indexed in Pubmed: 25851829.

26. Aschebrook-Kilfoy B, Schechter RB, Shih YCT, et al. The clinical and economic burden of a sustained increase in thyroid cancer incidence. Cancer Epidemiol Biomarkers Prev. 2013; 22(7): 1252-1259, doi: 10.1158/1055-9965.EPI-13-0242, indexed in Pubmed: 23677575.

27. Kandil E, Noureldine SI, Abbas A, et al. The impact of surgical volume on patient outcomes following thyroid surgery. Surgery. 2013; 154(6): 1346-52; discussion 1352, doi: 10.1016/j.surg.2013.04.068, indexed in Pubmed: 24238052.

28. Kiernan CM, Weiss VL, Mehrad M, et al. New terminology - noninvasive follicular neoplasm with papillary-like nuclear features (NIFTP) and its effect on the rate of malignancy at a single institution. Surgery. 2018; 163(1): 55-59, doi: 10.1016/j.surg.2017.04.041, indexed in Pubmed: 29126555. 